\title{
APPLYING HYPOPLASTIC MODEL FOR SOFT SOILS TO THE ANALYSIS OF ANCHORED SHEETING WALL
}

\author{
Tomáš KADLÍČEK, Tomáš JANDA and Michal ŠEJNOHA*
}

Department of Mechanics, Faculty of Civil Engineering, Czech Technical University in Prague, Thákurova 7 , 16629 Prague 6, Czech Republic

*Corresponding author's e-mail: sejnom@fsv.cvut.cz

\section{ARTICLE INFO}

Article history:

Received 30 July 2015

Accepted 12 October 2015

Available online 17 December 2015

Keywords:

Hypoplastic model

Soft soil

Anchored sheeting

Finite element simulations

\begin{abstract}
This paper is concerned with a practical application of the hypoplastic constitutive model for soft soils. In particular, two specific implementations, so called standard and the one based on smallstrain theory, are considered and the resulting predictions are compared with those provided by the Mohr-Coulomb model. As an example, we considered an anchored underground wall built during the construction of extension of the line A of Prague's subway. The results of all numerical simulations obtained with the help of Geo5 FEM software were also compared with in situ measurements with attention devoted mainly to the evolution of deformation of the underground wall and of pre-stress forces of rope anchors. It is shown that extended formulation of the hypoplastic model accounting for high stiffness of soil at very small strains should be adopted to arrive at reliable and meaningful predictions of the soil response.
\end{abstract}

\section{INTRODUCTION}

Apart from conventional constitutive models such as those based on the Mohr-Coulomb (MC) failure criterion (Potts and Zdravkovič, 1999) the models based on the theory of hypoplasticity (Mašín 2005) have been gaining constant attention supported by their predictive capability of the soil behavior particularly when ground settlements and underground deformations are of the primary interest. Unlike the former models the latter ones allow for capturing the actual nonlinear behavior of soils even at low loads that do not exceed their strength as well as during unloading. On the contrary, an extensive use of these models in practice, at least during the preliminary design, is precluded by the lack of data allowing for standard classification and the need for time consuming laboratory measurements and their numerical simulations to determine the necessary model parameters virtually for every soil.

This is not the case of models of the MC type which essentially rely on effective cohesion $\mathrm{c}_{\mathrm{ef}}$, effective angle of internal friction $\varphi_{\text {ef }}$ and Young's modulus of soil in loading and unloading, which are considered as standard outputs of laboratory measurements or can be pulled from a database based on their standard classification. However, these parameters are strictly valid for a certain range of stresses only. For the correct use it is thus the designer's responsibility to properly account for the range of stress to which the soil will be subjected. Application of different moduli for primary loading and unloading, their dependence on the actual stress level or extension to small strain theory may provide further improvements which together with its simplicity still keep it at the forefront of engineering interest.

Although some of the features of the soil behavior can directly be described by the hypoplastic model (HC), the material parameters that enter the model formulation are not so common in engineering practice. The $\mathrm{HC}$ model arises from the family of critical state models such as the Cam-Clay (CC) model (Roscoe and Burland, 1968). Similar to CC model the $\mathrm{HC}$ model distinguishes between material parameters and state variables. The material parameters include for example the slope of normal compression line $\lambda$ and the slope of swelling line $\kappa$ which directly relate to the slopes of the loading and unloading curves observed in oedometer, or the critical angle of internal friction $\varphi_{\text {ef }}$. These parameters are expected to be the same for soils of identical composition irrespective of stress state and the actual soil porosity. The state variables on the other hand characterize the current state of the soil. These include the soil porosity $\mathrm{n}$, the void ratio e or the overconsolidation ratio OCR. Unlike CC model the HC model does not, however, follow the classical plasticity theory. It abandons the concept of plastic surface and introduces the so called state boundary surface (SBS), where is similarly to the CC model drawn in the stress-porosity space and bounds all admissible states that the soil can experience during the loading process. The size of this surface is controlled by the current values of state variables which in turn evolve together with the current stress according to the stress-strain law formulated in the rate form (Janda, 2013). The soil is thus assumed to undergo inelastic deformations from the onset of 


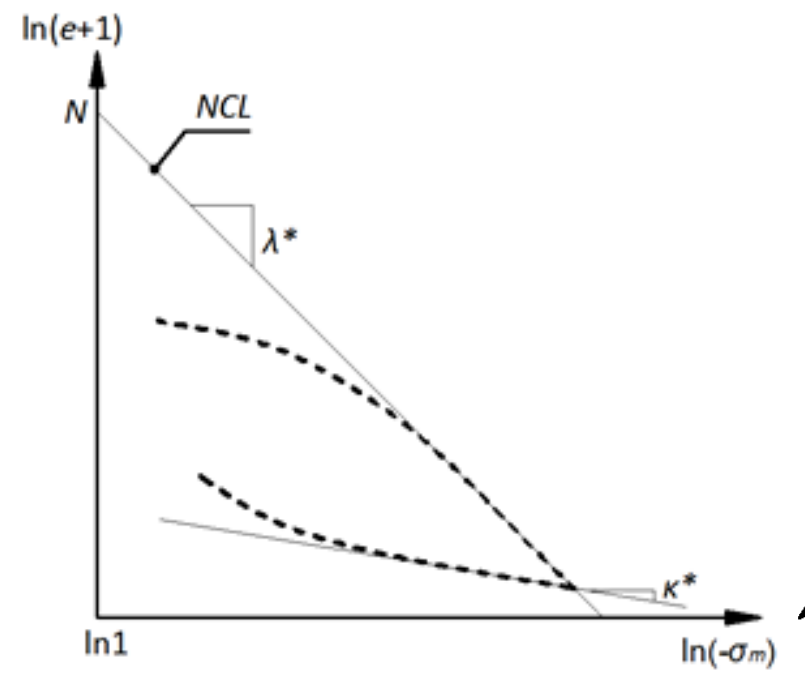

(a)

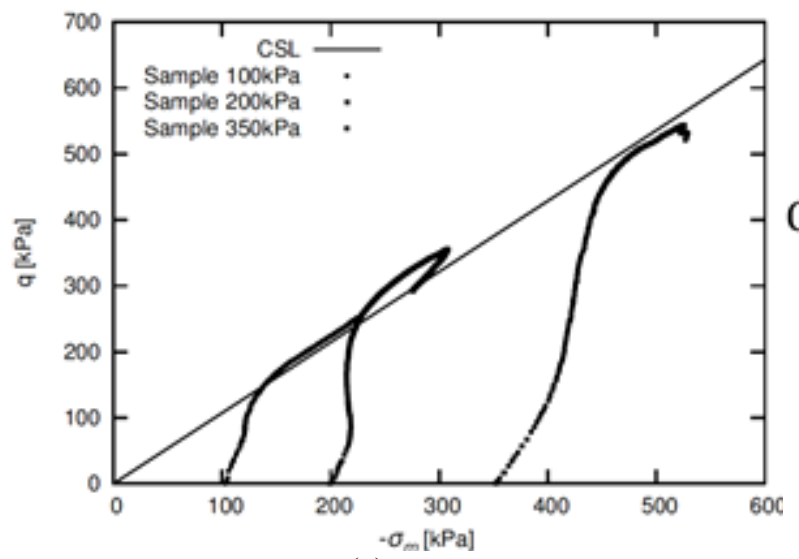

(c)

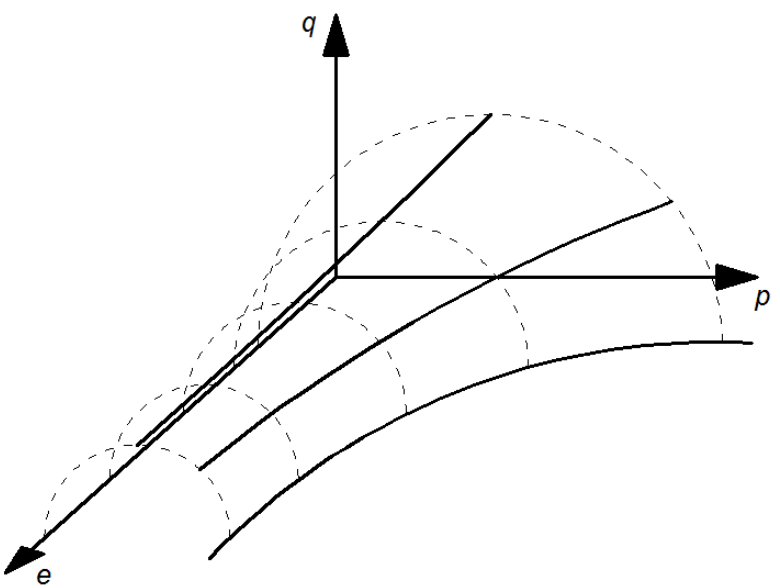

(b)

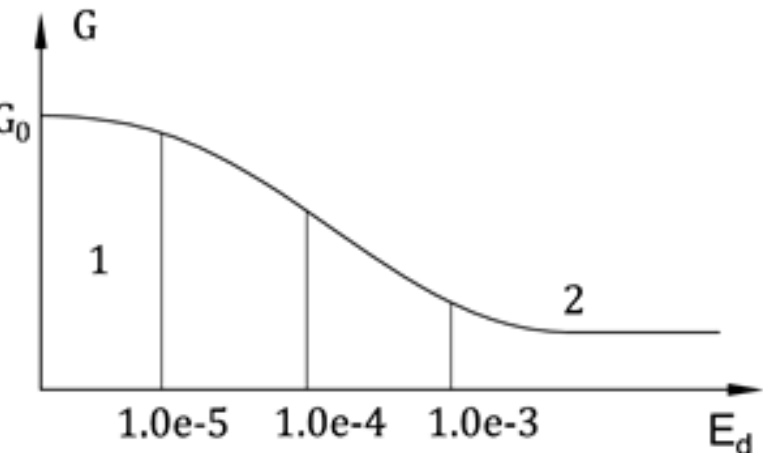

(d)

Fig. 1 Isotropic compression test in $\ln (e+1) \times \ln \left(-\sigma_{\mathrm{m}}\right)$ space, b) State Boundary Surface, c) Results of triaxial test plotted in $\left.-\sigma_{m} \times q, d\right)$ Degradation of shear modulus with increasing equivalent deviatoric strain

loading. The model well reflects the variation of soil stiffness on the degree of compaction, the current stress state as well as the loading direction, i.e. loading-unloading sequence. Apart from the standard formulation, which relies more or less on the same set of parameters as the $\mathrm{CC}$ model, the literature offers its extended version (HCE) for the description of the soil response at very small strains not exceeding 0.001 (Niemunis and Herle, 1997; Mašín, 2005).

As already mentioned, despite undoubted benefits of the HC model, its vast acceptance by engineering community is still lacking. This can partially be attributed to insufficient theoretical knowledge of this model but largely to the need for time consuming calibration (Janda and Šejnoha, 2013; Mašín, 2015). To overcome this burden a research project TA04031604 has been put forward with the aim of providing an automated tool for the determination of basic model parameters as well as of providing a database of these parameters for typical soils based on their standard classification. The same expectation is accorded to the papers aimed at practical use of HC models (Svoboda et al., 2009;
Svoboda et al., 2010; Kadlíček et al., 2015) including the present contribution, which is organized as follows. Following the introductory part attention is accorded to a brief description of basic features of the HC model in Section 2. Some basic information of the investigated construction site is provided in Section 3. The actual numerical analysis and achieved results are presented next in Sections 5 and 6. The essential findings are then summarized in Section 7. Some specific differences in the implementation of hypoplastic models and the Mohr-Coulomb model are briefly outlined in the appendix.

\section{FORMULATION OF HYPOPLASTIC MODEL}

The hypoplastic model is defined in its basic version by five parameters $\lambda^{*}, \kappa^{*}, N, \varphi_{\mathrm{cr}}$ and $\mathrm{r}$. Graphical representation of the first three parameters is evident from Figure 1(a) showing the plot of isotropic compression test. In the $\ln (e+1) \times \ln \left(-\sigma_{\mathrm{m}}\right)$ space, where e is the void ratio and $\sigma_{\mathrm{m}}$ represents the mean stress, the parameters $\lambda^{*}$ and $\kappa^{*}$ determine the slope of the Normal Compression Line (NCL) and the 
slope of the swelling line, respectively. There is an obvious difference between the HC model and the CC model, where NCL is defined in the $\mathrm{e} \times \ln \left(-\sigma_{\mathrm{m}}\right)$ space. A simple relationship can be derived to relate the corresponding sets of parameters of the $\mathrm{HC}$ and $\mathrm{CC}$ models for a selected stress range (Janda and Šejnoha 2013). The location of NCL in the $\ln (e+1) \times \ln \left(-\sigma_{\mathrm{m}}\right)$ space is specified by the parameter $\mathrm{N}$, which corresponds to the value of $\ln (e+1)$ for the value of the mean stress $\sigma_{m}=-1 \mathrm{kPa}$. All three parameters can be determined from both isotropic compression and oedometric tests. For the latter case, some extra work is needed to properly adjut the parameter $\mathrm{N}$ (Janda and Šejnoha, 2013; Mašín, 2015). The envelope of all possible states of soil is represented by the SBS displayed in Figure 1(b), where $\mathrm{q}$ stands for the equivalent deviatoric stress. A novel formulation which introduces a unique explicit SBS is presented in (Mašín, 2013). Notice that the yield surface of the CC model takes on a similar shape as the cross-section of the SBS (Potts and Zdravkovič, 1999).

Parameters $\varphi_{\text {cr }}$ and $r$ can be obtained from an undrained triaxial shear test. The determination of the critical angle of internal friction $\varphi_{\text {cr }}$ is rather straightforward and it follows from the slope of the line plotted in the $-\sigma_{\mathrm{m}} \times \mathrm{q}$ space that connects the origin with the value of the deviatoric stress $q$ corresponding to the critical state, see Figure 1(c). This state is characterized by the constant value of the volumetric strain $\varepsilon_{\mathrm{v}}$ while the axial $\varepsilon_{\mathrm{a}}$ or rather deviatoric strain components keep on increasing under continuous shearing. The parameter $r=K / G$ finally determines the ratio of the tangent bulk modulus at isotropic stress state $\mathrm{K}$ and the tangent shear modulus $\mathrm{G}$ which corresponds to an undrained triaxial test starting from this same isotropic stress. Its determination calls for a numerical simulation of the triaxial test allowing us to fit the parameter $r$ by comparing the computationally and experimentally derived curves plotted, e.g. in the $\mathrm{q} \times \varepsilon_{\mathrm{a}}$ space.

The basic version of the HC model can be extended to account for the observed high stiffness of soil when experiencing very small strains. In the literature, this is associated with the concept of small strain stiffness (Potts and Zdravkovič, 1999) or the concept of intergranular strain (Niemunis and Herle, 1997). Such a state is controlled by additional five parameters $\mathrm{R}, \beta, \mathrm{X}, \mathrm{m}_{\mathrm{R}}$ and $\mathrm{m}_{\mathrm{T}}$ This makes the use of hypoplastic model even more complicated as these parameters can no longer be determined from conventional laboratory tests. Instead, the application of so called bender elements and local sensors of deformation is needed to relate the degradation of the shear modulus $G$ to the value of the deviatoric strain $E_{d}$, see Figure 1(d).
The concept of intergranular strain assumes that the total strain is composed of a small elastic strain of a layer between the soil grains (intergranular strain) and the strain caused by a mutual shift and rotation of grains. The latter one sets in once the deviatoric strain $E_{d}$ exceeds its limiting value. The range of the elastic intergranular strain is controlled by the parameter $\mathrm{R}$. Parameters $\beta$ and $\chi$ control the degree of stiffness degradation with an increasing strain. These three parameters can again be delivered at best with the help of parametric study by matching the measured and calculated curves in the $\mathrm{G} \times \mathrm{E}_{\mathrm{d}}$ space, recall Figure 1(d). The last two parameters $\mathrm{m}_{\mathrm{R}}, \mathrm{m}_{\mathrm{T}}$ control the initial stiffness when changing the strain direction by $180^{\circ}$ and $90^{\circ}$, respectively. They receive the values of the multiple of shear stiffness of the basic model. Details of the calibration procedure for the soil material adopted in the present study and in (Kadlíček et al., 2015) are available in (Janda and Šejnoha, 2013). This approach provides the basis for an automating calibration process which is our present research effort (Project No. TA04031603 2014). For those interested in new developments in the field of intergranular strain we refer to a recent work on this subject by (Fuentes and Triantafyllidis, 2015).

\section{CONSTRUCTION SITE}

Samples of the soil used in the calibration of hypoplastic model discussed in the previous section were collected from the construction ditch E1, which was exploited to construct an access tunnel in the direction normal to the Evropská avenue, the principal artery connecting the Prague 6 district and the Václav Havel airport. Both the construction ditch and access tunnel were built as part of the extension of line A of Prague's subway excavated using the TBM technology. The access tunnel was built in advance and served later to pull the boring machines from the tunnel. Details of the analysis of the access tunnel can be found in (Kadlíček et al., 2015). Here, we limit our attention to the analysis of the construction ditch, the plane view of which is plotted in Figure 2, with emphases on the comparison of performance of the selected constitutive models.

The construction ditch is located between Kanadská and Velvarská streets next to the Evropská avenue heavily loaded by traffic. Thus the construction ditch as well as the access tunnel had to be built with caution not to allow for any damage to the Evropská avenue, which was opened to traffic during the whole construction time of the subway. The ditch plane is having a trapezoidal shape with dimensions of $31.4 \times 20.5 \mathrm{~m}$ and a depth of $20.3 \mathrm{~m}$. The walls of the ditch are constructed from steelreinforced concrete panels having thickness of $1 \mathrm{~m}$ and length of 2.5-6.7 $\mathrm{m}$ depending on their location. The two parallel walls ST1 and ST4, see Figure 2, were constructed with different foundation depth of $8.15 \mathrm{~m}$ and $6.15 \mathrm{~m}$, respectively. Transition between these 


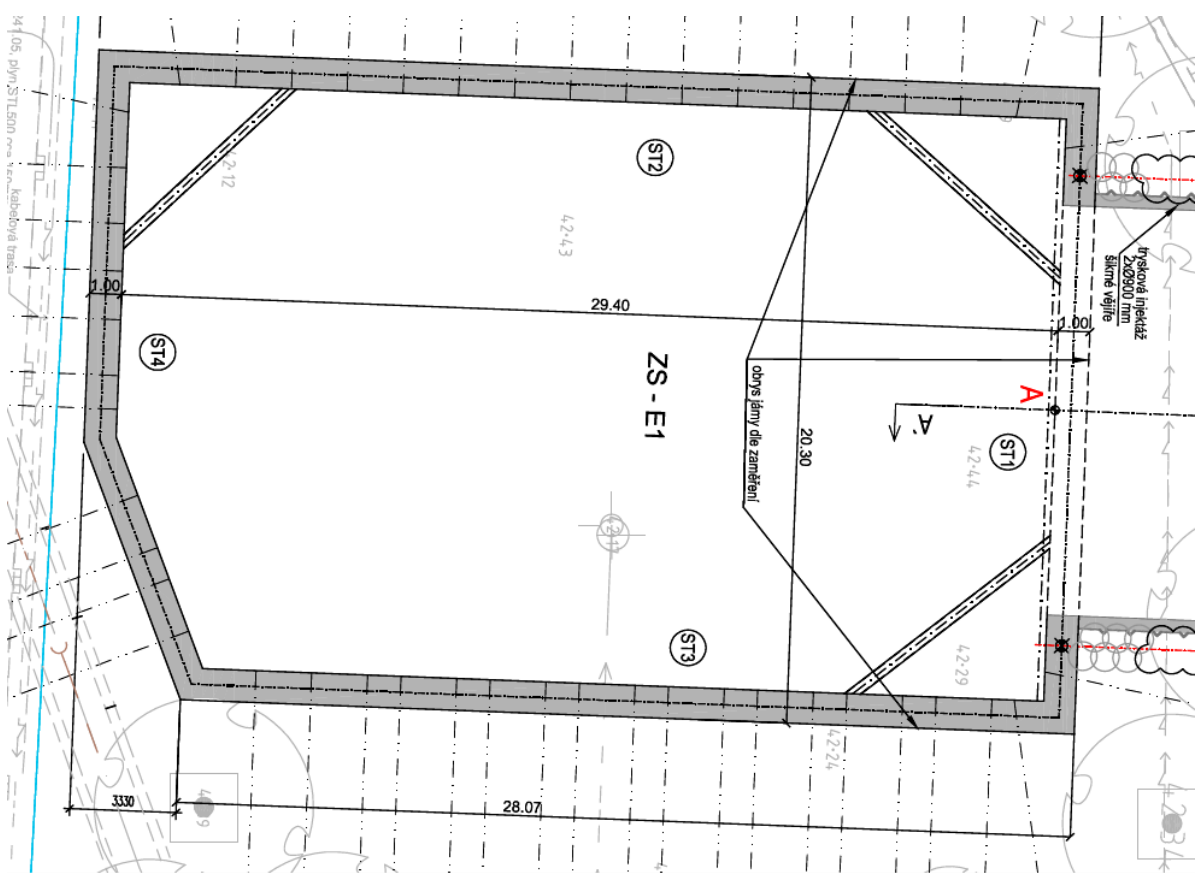

Fig. 2 Plane view of the construction site (METROPROJEKT Prague PLC 20092012).

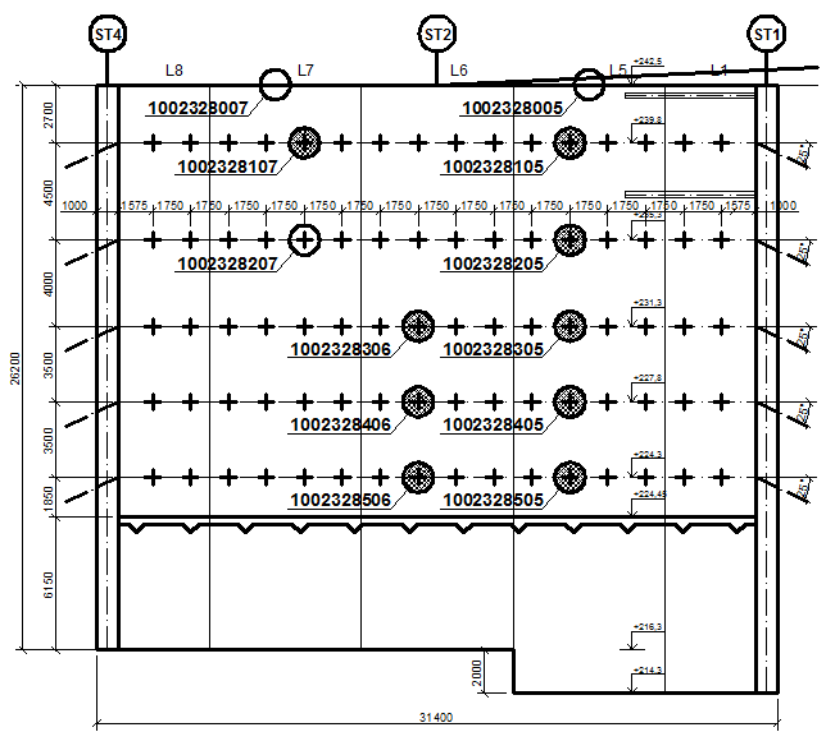

(a)

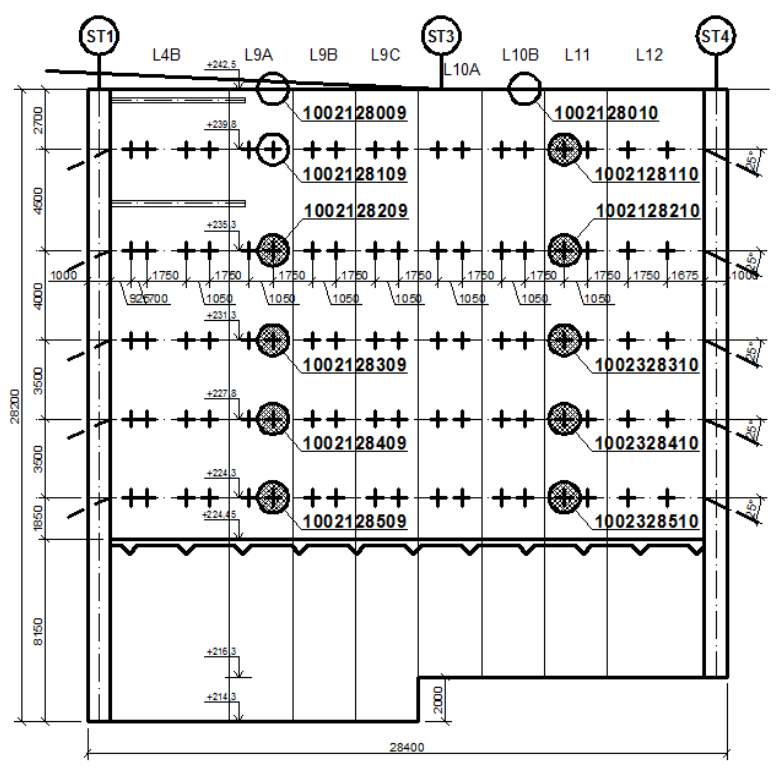

(b)

Fig. 3 Measurement points (white circle represents trigonometric point, dashed circle represents trigonometric point equipped with dynamometer: a) ST2 wall, b) ST3 wall.

two levels of foundation was accomplished via jump between the walls ST2 and ST3 as seen in Figure 3. The overall height of panels amounted to $26.2 \mathrm{~m}$ and $28.2 \mathrm{~m}$, respectively.

The walls were stabilized with the help of rope anchors and steel props placed in the corners of the ditch, recall Figure 2. Six and nine rope anchors were installed at five levels at the angle of $25^{\circ}$ spread by $1.5 \mathrm{~m}$ in the horizontal and $3.5 \mathrm{~m}$ in the vertical directions, respectively. As displayed in Figure 3 the walls were equipped with several measuring devices to allow for a continuous measurement of displacements during the construction process. To that end, trigonometric points were gradually mounted to the walls at six levels. Inclinometers were further used to measure the mutual inclination of the walls ST2 and ST3. Apart from that, dynamometers were located irregularly at each of the anchor depth to monitor a gradual evolution of anchor forces. The basic characteristics of anchors are summarized in 
Table 1 Basic characteristics of rope anchors.

\begin{tabular}{c|c|c|c|c}
\hline Row & Inclination $\left[{ }^{\circ}\right]$ & Length $[\mathrm{m}]$ & Cross-section area $\left[\mathrm{mm}^{2}\right]$ & Anchor force $\mathrm{F}_{c}[\mathrm{kN}]$ \\
\hline 1 & 25 & 31 & 840 & 835 \\
2 & 25 & 29 & 840 & 835 \\
3 & 25 & 29 & 1120 & 750 \\
4 & 25 & 26 & 1120 & 940 \\
5 & 25 & 22 & 1120 & 870 \\
\hline
\end{tabular}

Table 1. The pre-stress forces in this table represent averages of the actual forces introduced at individual levels.

\section{MATERIAL DATA}

The principal objective of this contribution is to compare the predictive capability of the classical Mohr-Coulomb type of constitutive model with those based on the theory of hypoplasticity. Site investigations suggested three particular types of soil to be considered in the analysis, see ahead Figure 4 . The MC model was considered for all types of soils whereas the two types of hypoplastic models were adopted for the layer of sediments only. The corresponding material data were established based on the calibration procedure described in Section 2 and in (Janda and Šejnoha, 2013).

The necessary laboratory measurements including CIUP triaxial test (isotropic consolidated undrained test with pore pressure measurement) and oedometric test for the layer of sediments were performed by Arcadis CZ, ltd. and at the Faculty of Science of the Charles University in Prague. Triaxial and oedometric tests allowed also for the determination of effective cohesion $\mathrm{c}_{\mathrm{ef}}$ and effective angle of internal friction $\varphi_{\text {ef }}$ needed in the MC model. Young's modulus was derived from the measured oedometric modulus adopting the Poisson ratio $v=0.37$, see Table 2 . This value was taken from the Czech standards ČSN 731001 (ČSN731001 1987) and approximately corresponds to the value of $v=\mathrm{K}_{0} /\left(1+\mathrm{K}_{0}\right)$, where the coefficient of lateral earth pressure $\mathrm{K}_{0}$ follows from the Jaky formula $\mathrm{K}_{0}=1-\sin \varphi_{\mathrm{ef}}$. Owing to the fact that during excavation the ground soil experiences unloading rather than primary loading the Young modulus was estimated from the unloading branch of the oedometric test. The same reasoning was also applied to the layer of landfill. Point out that the material data of the MC model for the layer of landfill and the layer of slate were not measured experimentally but instead pulled from the available database based on the classification according to the Czech standards ČSN 731001 (ČSN731001 1987). The resulting material data employed in the numerical analysis are listed in Tables 2-4.

Table 2 Material parameters of Mohr-Coulomb model.

\begin{tabular}{l|c|c|c|c|c|c|c}
\hline Soil & $\begin{array}{c}\mathrm{E} \\
{[\mathrm{MPa}]}\end{array}$ & $\begin{array}{c}v \\
{[-]}\end{array}$ & $\begin{array}{c}c_{e f} \\
{[\mathrm{kPa}]}\end{array}$ & $\begin{array}{c}\varphi_{e f} \\
{\left[{ }^{\circ}\right]}\end{array}$ & $\begin{array}{c}\gamma \\
{\left[\mathrm{kN} / \mathrm{m}^{3}\right]}\end{array}$ & $\begin{array}{c}\gamma_{\text {sat }} \\
{\left[\mathrm{kN} / \mathrm{m}^{3}\right]}\end{array}$ & $\begin{array}{c}\text { Thickness } \\
{[\mathrm{m}]}\end{array}$ \\
\hline Landfill & 12 & 0.28 & 2 & 35.5 & 20 & 21 & 2.8 \\
Sediments & 56.5 & 0.37 & 20 & 30 & 20 & 22 & 17.7 \\
Slate & 40 & 0.30 & 25 & 23 & 23 & 24 & 40 \\
\hline
\end{tabular}

Table 3 Material parameters of hypoplastic model.

\begin{tabular}{c|c|c|c|c|c}
\hline \multirow{2}{*}{ Soil } & $\lambda^{*}$ & $\kappa^{*}$ & $\mathrm{~N}$ & $\varphi_{c r}$ & $r$ \\
& {$[-]$} & {$[-]$} & {$[-]$} & {$\left[{ }^{\circ}\right]$} & {$[-]$} \\
\hline Sediments & 0.051 & 0.0078 & 0.677 & 28.4 & 0.2 \\
\hline
\end{tabular}

Table 4 Material parameters controlling evolution of intergranular strain.

\begin{tabular}{c|c|c|c|c|c}
\hline \multirow{2}{*}{ Soil } & $\mathrm{R}$ & $m_{R}$ & $m_{T}$ & $\beta_{r}$ & $\xi$ \\
& {$[-]$} & {$[-]$} & {$[-]$} & {$[-]$} & {$[-]$} \\
\hline Sediments & 0.0001 & 9.3 & 9.3 & 0.2 & 1 \\
\hline
\end{tabular}




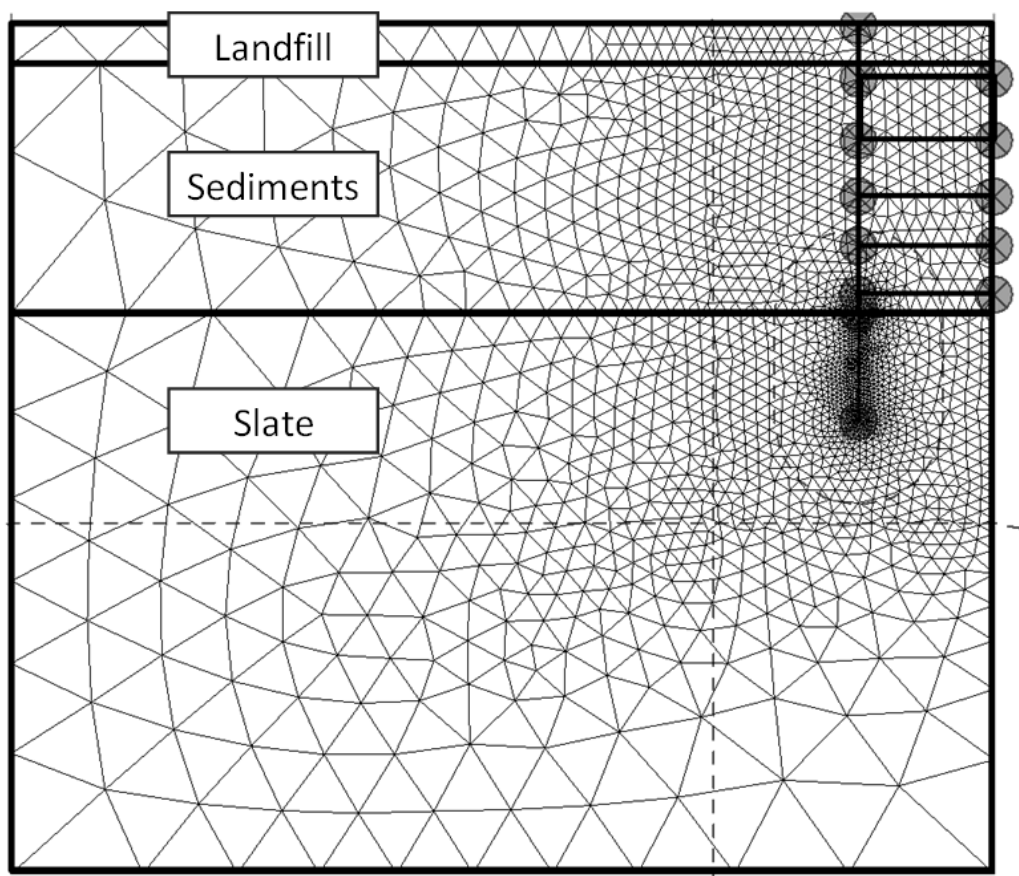

Fig. 4 Geometrical model and finite element mesh.

\section{NUMERICAL ANALYSIS}

The numerical analysis was carried out using the Geo5 FEM finite element software (Fine-Ltd. 2015). It makes possible to solve a two-dimensional problem under plane strain conditions, which in our case corresponds to the analysis of an infinite wall with zero out-of-plane displacements. Therefore, also the anchors are considered per $1 \mathrm{~m}$ run replacing individual anchors with given dimensions by an equivalent membrane. Since the actual construction ditch is three-dimensional, we may expect some inconsistencies in the predictions and in situ measurements as will be seen in Section 6. To eliminate these discrepancies, the geometry of walls ST2 and ST3 was considered in the formulation of geometrical model as these walls are the most longest ones and they are least affected by additional reinforcing elements and various jumps unable to reflect in $2 \mathrm{D}$ simulations.

\subsection{GEOMETRY AND FINITE ELEMENT MESH}

To simplify the analysis the structure was assumed symmetric making possible to consider only one half of the structure. The resulting computational model showing individual soil layers, position of the wall and assumed excavated layers appears in Figure 4. To eliminate the influence of boundary conditions the outer boundaries were located sufficiently far from the excavated region rendering a $70 \mathrm{~m}$ high and a $60 \mathrm{~m}$ wide model.

Generation of the finite element mesh was driven by two competing issues namely the computational efficiency and accuracy. To that end, a sufficiently fine mesh was built in the prominent region close to the wall being represented by beam elements of the
Mindlin type. This was also promoted by the use of contact elements along the soil-wall interface where mesh refinement may help to stabilize the convergence process. Smooth convergence was further supported by properly choosing the elastic normal $\mathrm{K}_{\mathrm{n}}$ and shear $\mathrm{K}_{\mathrm{s}}$ stiffnesses of this element. Shearing along the soil-wall interface was governed by the Mohr-Coulomb law with excluded tension. Table 5 stores the associated material data with $\mathrm{c}, \mu, \mathrm{R}_{\mathrm{t}}$ standing for the cohesion, the coefficient of friction and the tensile strength, respectively. Since the objective of this work is to promote applicability of the $\mathrm{HC}$ model rather than to provide an accurate prediction of the wall response and because no measurements of interfacial friction parameters were carried out we chose the values of $c, \mu, R_{t}$ such as to give relative displacements between the soil and wall not too far from the measured values.

On the contrary, the region far from the wall was covered by a coarser mesh. This not only reduced the computational burden but allowed for better distribution of anchor forces into the soil. Note that anchors are modeled as two point rod elements not directly associated with the nodes of an underlying mesh but rather linked to the degrees of freedom of a tying object; the plane element representing soil and the beam element representing wall in particular. Recall the basic geometrical data of anchors in

Table 5 Material parameters of contact element.

\begin{tabular}{c|c|c|c|c}
\hline $\begin{array}{c}\mathrm{K}_{\mathrm{n}} \\
{\left[\mathrm{kN} / \mathrm{m}^{3}\right]}\end{array}$ & $\begin{array}{c}\mathrm{K}_{\mathrm{s}} \\
{\left[\mathrm{kN} / \mathrm{m}^{3}\right]}\end{array}$ & $\begin{array}{c}\mathrm{c} \\
{[\mathrm{kPa}]}\end{array}$ & $\begin{array}{c}\mu \\
{[-]}\end{array}$ & $\begin{array}{c}\mathrm{R}_{\mathrm{t}} \\
{[\mathrm{kPa}]}\end{array}$ \\
\hline 1000000 & 3000 & 10 & 0.7 & 0 \\
\hline
\end{tabular}




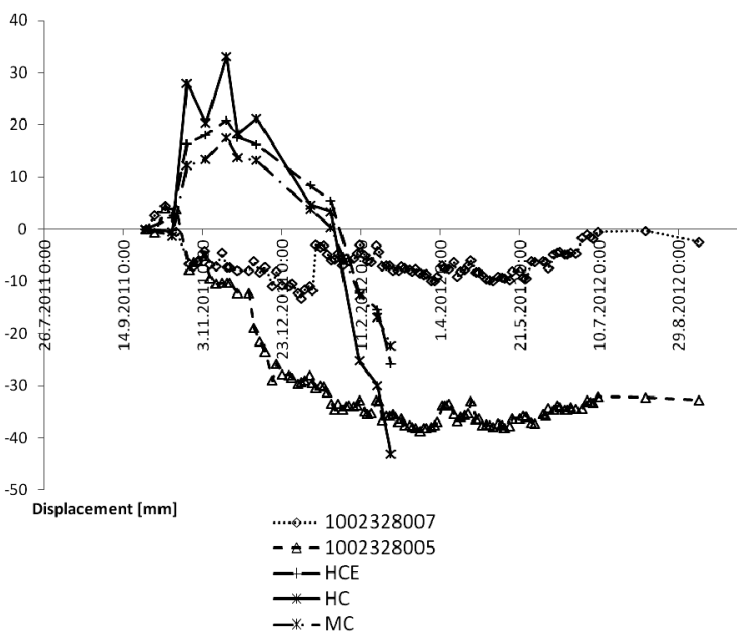

(a)

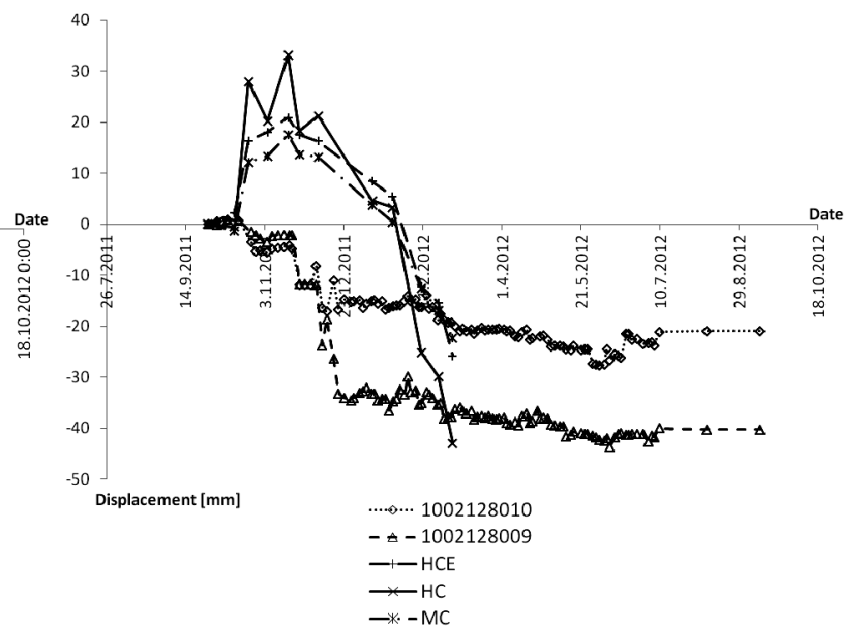

(b)

Fig. 5 Time evolution of horizontal displacements at the top of the wall: a) ST2 wall, b) ST3 wall. Comparison of displacements measured at selected trigonometric points with numerical predictions.

Table 1. Here, the Young modulus equal to $190 \mathrm{GPa}$ was assigned to all anchors. The material properties of a $1 \mathrm{~m}$ thick wall corresponding to concrete C35/45 were taken from the program database.

\subsection{MODELING CONSTRUCTION STAGES}

The analysis attempted to follow the actual construction as close as possible. This was achieved by splitting the building process into several calculation stages simulating individual excavation steps and installation of anchors.

The first stage served to generate the initial geostatic stress. In this calculation step all soils were assigned the MC model owing to the fact that the $\mathrm{HC}$ model is defined for the compression region only and the associated analysis cannot start from zero stresses. Underground water was also introduced by leveling the GWT with terrain. When the HC model was adopted for the layer of sediments, the second stage served to replace the MC model with the HC model. In this stage, the beam and interface elements were also introduced and the 1st excavation step was carried out. Prior to each excavation step the water level was adjusted to be aligned with new terrain boundary at the bottom of the ditch. This excavation stage was followed by the introduction of the 1st row of pre-stressed anchors. These two steps were repeated up to the final excavated depth of $20.5 \mathrm{~m}$. The anchors were attached to the beam elements one meter above the current depth of the construction ditch and pre-stressed to the value of force listed in Table 1. The entire analysis thus amounted to 13 calculation stages with advancing excavation steps up to the depth of $3.8,8.2,12.2,15.7,19.2$ and $20.5 \mathrm{~m}$, respectively.

Although experimental measurements on samples of sediments classified this soil as impermeable, the drained conditions were assumed for all layers of soils thus not accounting for potential evolution of excess pore pressure. This was partially driven by the fact that the excavation process was relatively slow. Also, no critical conditions, which might arise with undrained analysis assuming fast excavation, were observed in reality. The results are evaluated in the next section.

\section{RESULTS}

To compare the performance of individual constitutive models we make advantage of the results of an extensive monitoring program that provided almost a continuous evolution of horizontal displacements and anchor forces at several monitoring points. From the recorded time variation of anchor forces it was possible to determine the time of anchor installation. Although the analysis was time independent, it was possible to associate these time variations of both displacements and anchor forces with those derived numerically from the staged analysis described in the previous section.

\subsection{EVOLUTION OF HORIZONTAL DISPLACEMENTS}

Clearly, the points at the top of the wall were measured for the longest time. The data collected at these points were therefore selected for comparison with numerical predictions. Apart from that, the final deflection of the wall at the end of the construction process was also compared.

Figure 5 shows time evolution of horizontal displacements at four trigonometric points located at the top of the walls ST2 (points labeled as 1002328005 and 1002328007) and ST3 (points labeled as 1002128009 and 1002138010), recall Figure 3. It is easy to see that the variation of displacements at points both on the sides of the two parallel walls and at the points on the same wall very considerably. Both walls experience larger deflection in the locations close to the wall ST1 thus at the 


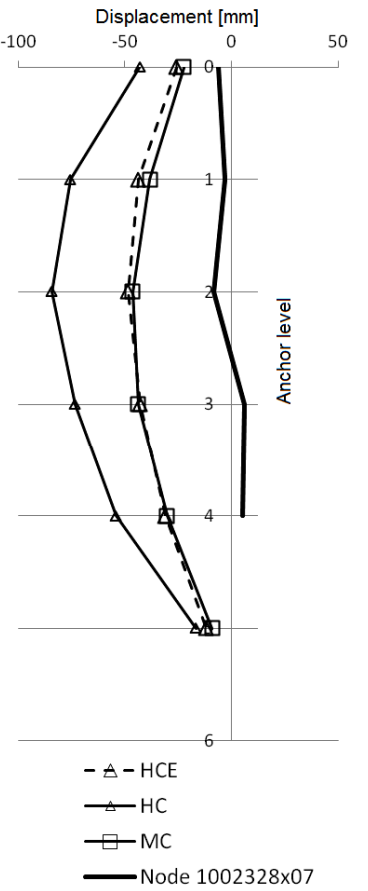

(a)

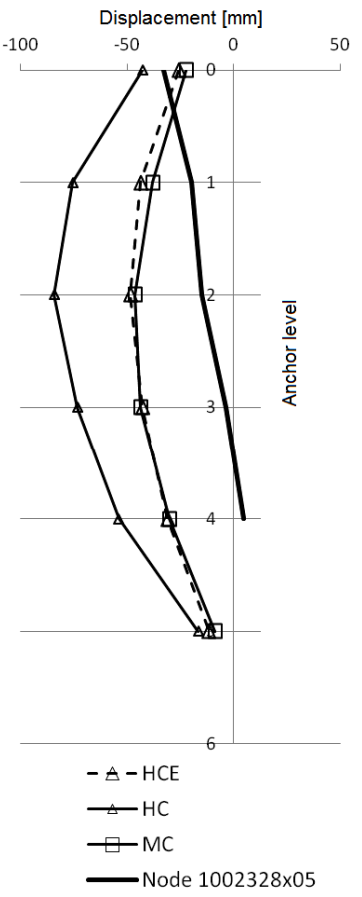

(b)

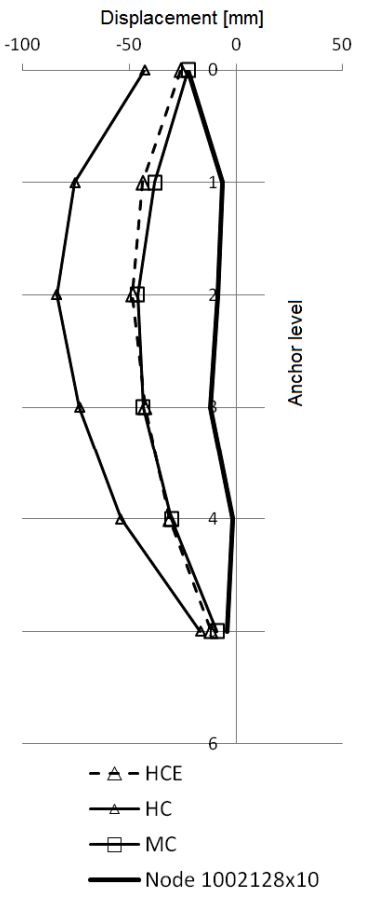

(c)

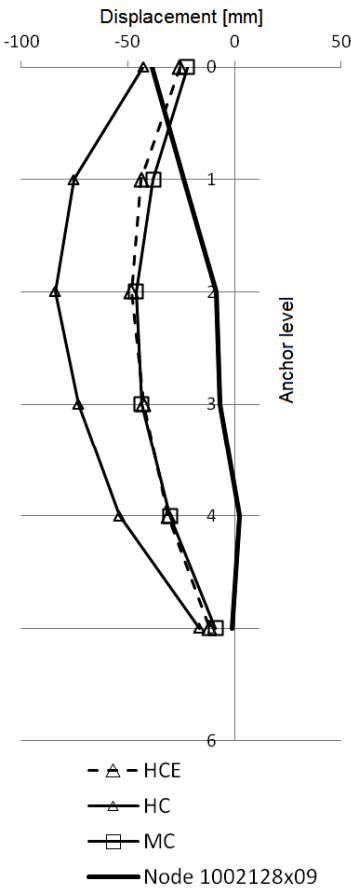

(d)

Fig. 6 Evolution of final horizontal displacements along of the wall: (a,b) ST2 wall, (c,b) ST3 wall. Comparison of displacements measured at selected trigonometric points with numerical predictions.

entrance to the tunnel. This confirms a threedimensional character of deformation of the examined construction ditch and at the same time makes difficult to compare these measurements with the results of numerical simulations. Point out that the assumed plane strain analysis and geometrical symmetry of the ditch yield a single value of the displacement at a given point for the entire structure. The resulting distributions associated with the use of different constitutive models are also plotted. Only the values obtained at the end of each calculation stage are shown starting with the second stage. Although linked to the actual time of construction they arise from a time independent analysis, whereas the measured displacements are also partly linked to the pore water pressure dissipation.

The initial distributions of the predicted displacements are characterized by a considerable evolution of positive displacements resulting from pushing the wall into the soil. This trend is not observed in reality for neither of the two examined walls. Despite the fact that the two-dimensional analysis seems inadequate it still may provide qualitative information as to the performance of individual models. It is seen a significant deviation of the predictions provided by the $\mathrm{HC}$ model in comparison to MC and HCE models. The HC model is evidently much more compliant resulting also into a hedgehog-like variation of the top wall deflection from stage to stage. The other two models on the other hand perform similarly with the final value falling into the range of the measured displacements.
Further comparison is offered by plotting the final distributions of the horizontal deflections along the wall in Figure 6. Although all measured deflections suggest bending of walls into the construction ditch, they again show considerable differences and promote already observed threedimensional character of the ditch response. Not surprisingly, the predicted shape of the deformed wall rather deviates from the actual one. However, the predictions of individual models are consistent further confirming an unrealistic compliance of the $\mathrm{HC}$ model.

\subsection{EVOLUTION OF ANCHOR FORCES}

One possible explanation to the deviation of the predicted and measured shape of wall deformation is an erroneous modeling of contribution of anchors. To reconcile this we plotted in Figure 7 the time evolution of averaged values of anchor forces for each row separately and again for both examined walls. These were then compared with predicted distributions pertinent to individual constitutive models.

Although numerical analyses assumed the values of real anchor forces, their values at the onset of action experience a certain drop in comparison to the measured values. This trend is particularly evident for the 1 st and the 2nd row located in the layer of sediments and can be blamed on ongoing consolidation process in less permeable sediments. Note that this behavior was not considered in numerical simulations as these assumed drained 


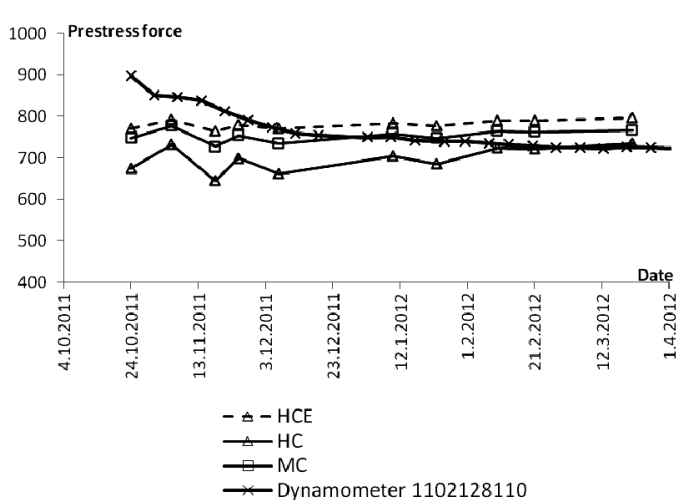

(a)

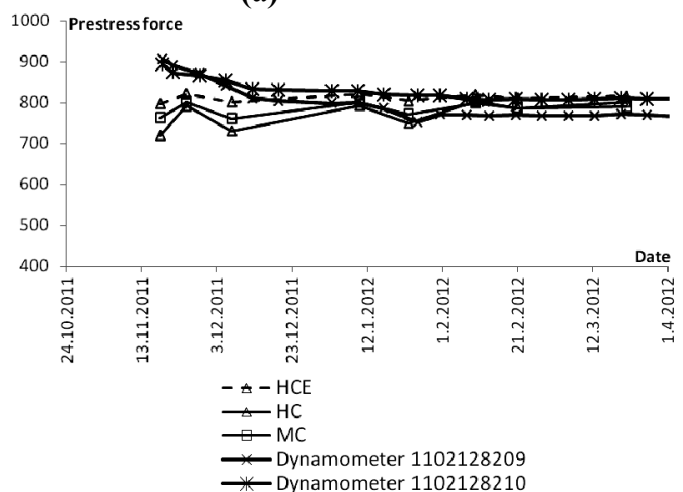

(c)

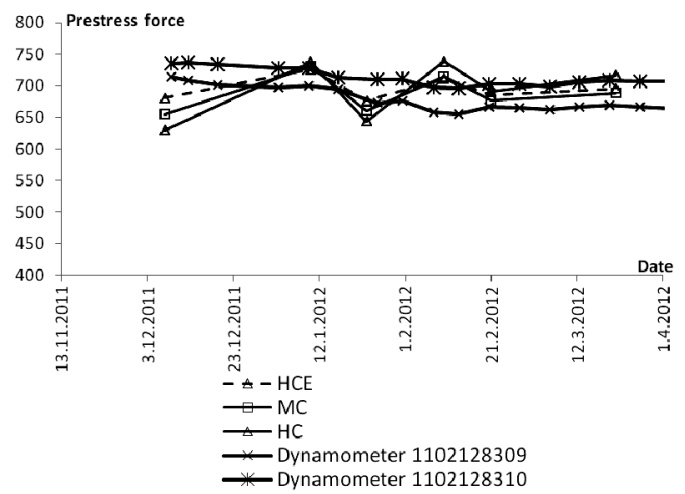

(e)

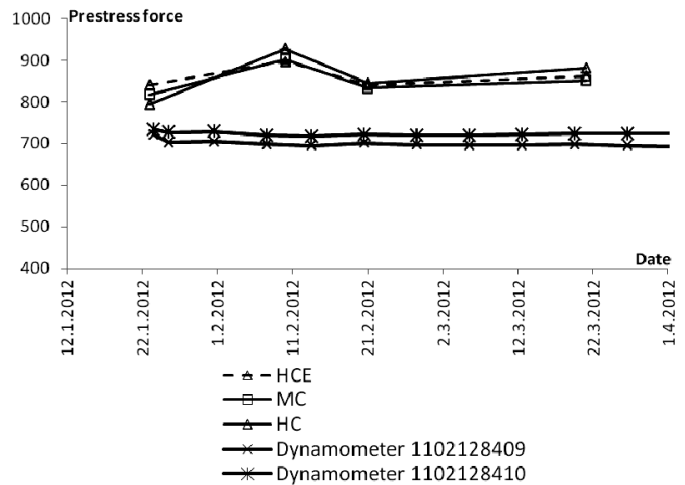

(g)

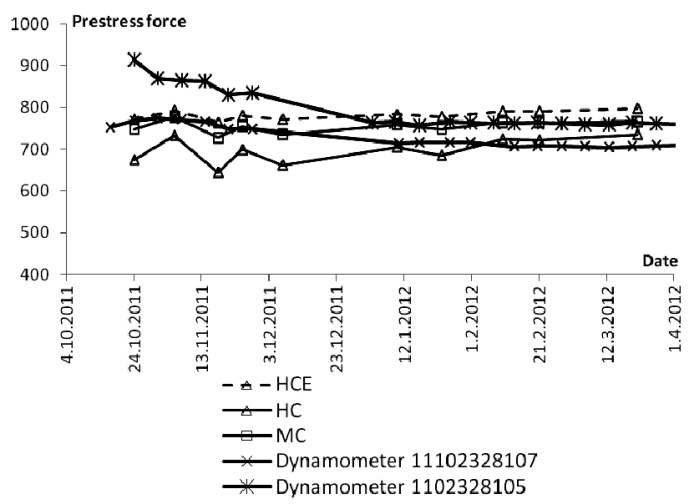

(b)

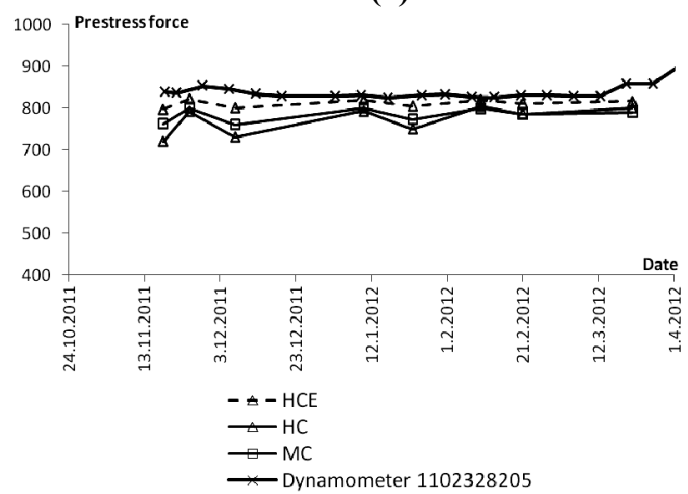

(d)

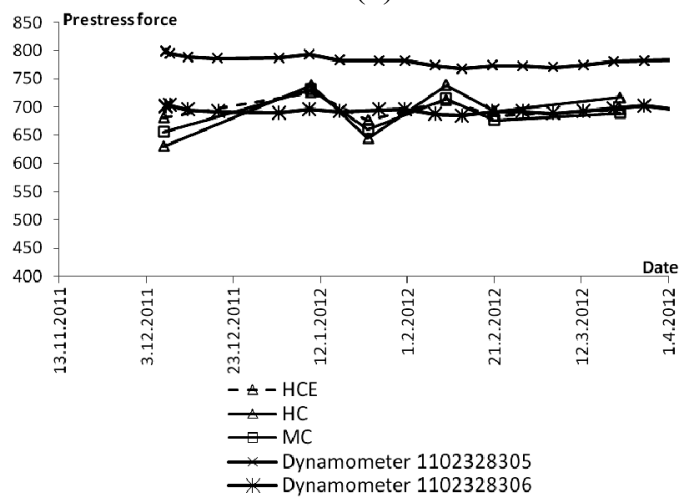

(f)

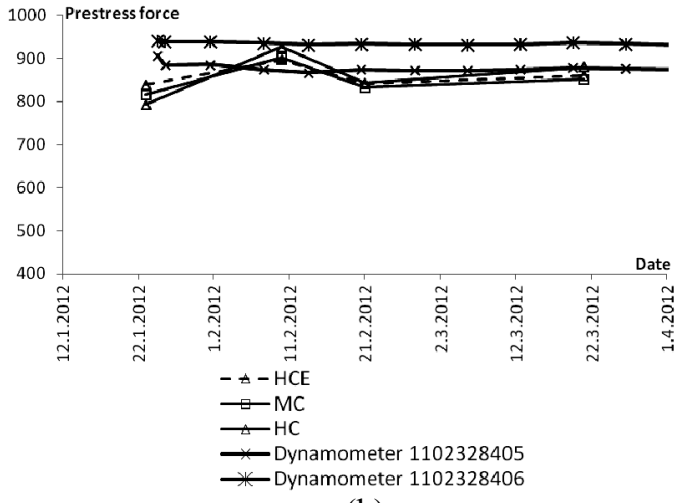

(h)

Fig. 7 Time variation of anchor forces: $(a, c, e, g)$ ST2 wall, (b,d,f,h) ST3 wall. Comparison of measured anchor forces with numerical predictions for four rows of anchors. 
conditions, which may result in an instantaneous reduction of the initial pre-stress force. In case of the 1st row of anchors this amounts to more than $100 \mathrm{kN}$. Further contribution to this effect from the numerical analysis can be associated with an insufficient anchorage at the anchor end point fixed into the soil body.

The next rows of anchors were already found in the layer of slate. Owing to a jointed character of this layer the effect of consolidation can be excluded. This is supported by the time variation of actual anchor forces in this layer, which shows more or less constant variation without noticeable oscillations. Apart from the wall ST2 and the 4th row of anchors the distributions of measured and calculated forces are reasonably close.

In general, the distributions of anchor forces predicted by MC and HCE models are comparable, whereas the results associated with the HC model suggest again deficiency in its predictive capability for the present set of material data. This is evident particularly for the first two layers of anchors mounted to the layer of sediments. The remaining rows of anchors mounted to a relatively stiff layer of slate were already not affected by the choice of the model for the layer of sediments.

\section{CONCLUSIONS}

The present paper was concerned with the comparison of predictive capabilities of hypoplastic models and the Mohr-Coulomb model as a common representative of models used in engineering practice. As an example we considered a sheeted construction ditch built to allow for an excavation of a technological tunnel used during the drilling of an extension line A of Prague's subway.
The results of numerical modeling clearly showed limitations of two-dimensional modeling, where relatively small dimensions of the ditch strengthened by various reinforcing elements on the one hand and weakened by the tunnel portal on the other created conditions far from those assumed in the plane-strain analysis. Arriving at more accurate predictions would call for a full three-dimensional model accompanied by the solution of a fully coupled time dependent consolidation analysis. Nevertheless, the present example was still sufficient to draw comparison primarily between the two versions of the hypoplastic model. Clear message when adopting a hypoplastic model is the need for incorporating the small strain stiffness concept as was the case of HCE model. Even when lacking data derived experimentally for a given soil sample it is still recommended to use the advanced formulation and employ some typical values for intergranular strain parameters (Mašín et al., 2006; Mašín, 2015).

When comparing the predictions delivered by the MC model with those provided by the HCE model one may argue the need for more advanced models. Here, it is necessary to mention the lack of stiffness evolution of the MC model as a function of stress, which is inherently hidden in the theory of hypoplasticity or the Cam-Clay models as its predecessor. Here, the qualitatively similar results both in terms of displacements and anchor forces were achievable only when considerably increasing the elastic stiffness in the MC model using Young's modulus for unloading rather than for primary loading for the layer of sediments. As a rule of thumb one may thus give preference to the hypoplastic model when settlements or in general displacements play the main role, whereas the Mohr-Coulomb type model appears sufficient in the modeling of shear failure.

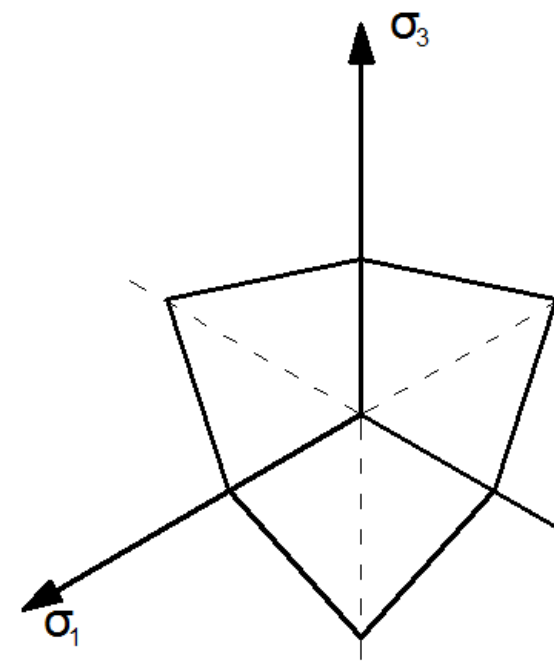

(a)

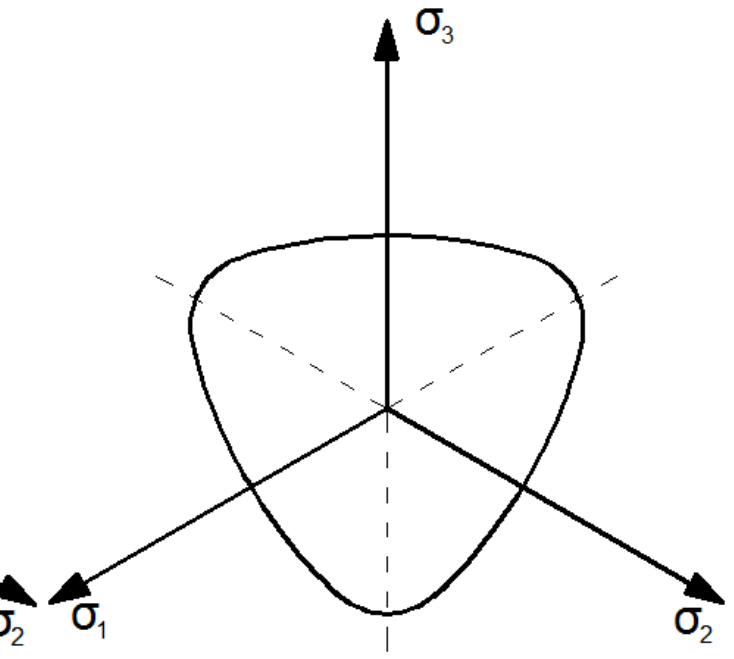

(b)

Fig. 8 Projection of the (a) Mohr-Coulomb yield surface and (b) State Boundary Surface into the deviatoric stress plane. 


\section{ACKNOWLEDGMENTS}

The financial support by the Technology Agency of the Czech Republic TAČR project No. TA04031603 and by the Project No. SGS15/031/OHK1/1T/11 is gratefully acknowledged.

\section{APPENDIX}

For the sake of completeness we give a brief outline of essential differences in implementation of the two types of compared constitutive models.

\section{MOHR-COULOMB MODEL}

The rate form of constitutive equations reads

$\dot{\sigma}=\mathbf{L}^{\mathrm{ep}} \dot{\varepsilon}$,

where $\mathbf{L}^{\mathrm{ep}}=\mathbf{L}^{\mathrm{ep}}\left(\mathrm{E}, \nu, \sigma, \mathrm{c}_{\mathrm{ef}}, \varphi_{\mathrm{ef}}, \psi\right)$ is the instantaneous or elasto-plastic stiffness matrix and $\boldsymbol{\sigma}$ and $\boldsymbol{\varepsilon}$ represent the stress and strain vectors, respectively. Incremental form of Eq. (1) is provided by

$\Delta \sigma=\mathbf{L}^{e l} \Delta \varepsilon+\Delta \lambda$,

where $\mathbf{L}^{\mathrm{el}}$ is the elastic stiffness matrix and the increment of eigenstress vector $\Delta \lambda$ follows from the flow rule

$\Delta \lambda=-\mathbf{L}^{e l} \Delta \mu$

$\Delta \mu=\varepsilon^{\mathrm{p} 1} \frac{\mathrm{m}}{\|\mathrm{m}\|}, \quad \mathrm{m}=\frac{\partial \mathrm{G}(\sigma, \psi)}{\partial \sigma}$

where $\varepsilon^{\mathrm{pl}}$ is the plastic multiplier, the vector $\mathbf{m}$ sets the direction of the plastic strain increment and $G$ stands for the plastic potential being function of stress and the angle of dilation $\psi$. In all present calculations $\psi=0$ was assumed. The value of plastic multiplier depends on the selected type of yield function, which in case of the Mohr-Coulomb model plots as an irregular hexagon in the deviatoric stress plane, see Figure 8 (a). Typically, the classical elastic predictorplastic corrector procedure combined with some sort of return mapping algorithm is employed to keep the stress point on the yield surface upon plastic loading.

\section{HYPOPLASTIC MODEL}

We limit our attention to the standard formulation, i.e. the $\mathrm{HC}$ model, and write the rate form of constitutive equations as

$\dot{\sigma}=\mathrm{L} \dot{\varepsilon}+\dot{\lambda}$,

where $\mathcal{L}=\mathcal{L}\left(\sigma, \lambda^{*}, \kappa^{*}, \varphi_{\mathrm{cr}}, \mathrm{r}\right)$ is the instantaneous elastic stiffness matrix depending on the current level of stress and material parameters of the hypoplastic model. The rate of eigenstress vector $\lambda$ provided by

$$
\dot{\lambda}\left(\sigma, \lambda^{*}, \kappa^{*}, \varphi_{\mathrm{cr}}, \mathrm{N}, \mathrm{e}\right)=-\mathrm{L} \dot{\mu}=-\mathrm{L}\|\dot{\varepsilon}\| \mathrm{Y} \frac{\mathrm{m}}{\|\mathrm{m}\|},
$$

is also a function of the current state of soil represented here by the void ratio e. Comparing the two pairs of Eqs. (1), (5) and (3), (6) we notice a formal similarity between the two models. The scalar $\|\dot{\varepsilon}\| \mathrm{Y}$ can be identified with the plastic multiplier $\boldsymbol{\varepsilon}^{\mathrm{pl}}$ and the vector $\mathbf{m}$ defines again the direction of the nonlinear strain rate increment $\mu$. The term $Y$ is a function of the 3 rd stress invariant and defines the shape of SBS in the deviatoric plane, see Figure 8(b).

The principal difference between the two models is that in case of hypoplasticity the soil behaves nonlinearly both in loading and unloading (the rate of eigenstress vector $\lambda$ is active at all times) whereas in case of plasticity the plastic strains $\mu$ in Eqs. (2)-(4) evolve only when the material point undergoes plastic loading and upon unloading the soil response is elastic. Also point out the way of integrating Eq. (5) to arrive at the current stress level for the current increment of strain. Unlike plasticity a direct forward integration of Eq. (5) is performed employing the Runge-Kutta method with a suitable order combined with a certain substepping procedure to minimize the integration error. Further details are available in (Mašín, 2005; Janda, 2013).

\section{REFERENCES}

ČSN731001: 1987, Building foundation, Ground beneath spread footing. CNI, Praha, (Czech standard).

Fine-Ltd.: 2015, GEO 5 MKP. GEO 5 Tunnel, http://www.fine.cz/.

Fuentes, W. and Triantafyllidis, Th.: 2015, ISA model: A constitutive model for soils with yield surface in the intergranular strain space. A constitutive model for soils with yield surface in the intergranular strain space, International Journal for Numerical and Analytical Methods in Geomechanics, 39 (11), 12351254. DOI: $10.1002 /$ nag. 2370

Janda, T.: 2013, Hypoplastic model for clays: Issues specific to the finite element implementation. Topping, B., Ivanyi, P. (Eds.): Proceedings of the Fourteenth International Conference on Civil, Structural and Environmental Engineering Computing, Civil-Com.

Janda, T. and Šejnoha, M.: 2013, Calibration of advanced material models for fine-grained soils using MKP software. Geotechnika, 2, 3-13, (in Czech).

Kadlíček, T., Janda, T., Šejnoha, M., Kuňák, J. and Urbánková, M.: 2015, Application of hypoplastic model of fine-grained soils in numerical modelling of natm tunnelling. Tunel, 24 (2), 69-82.

Mašín, D.: 2005. A hypoplastic constitutive models for clays. International Journal for Numerical and Analytical Methods in Geomechanics, 29, 311-336. DOI: $10.1002 /$ nag.416

Mašín, D.: 2013, Clay hypoplasticity with explicitly defined asymptotic states. Acta Geotechnica, 8 (5), 481-496. DOI: $10.1007 / \mathrm{s} 11440-012-0199-\mathrm{y}$

Mašín, D.: 2015, Hypoplasticity for Practical Applications. (https://web.natur.cuni.cz/uhigug/masin/hypocourse).

Mašín, D., Tamagnini, C., Viggiani, G. and Costanzo, D.: 2006, Directional response of a reconstituted engrained soil - part ii: performance of different 
constitutive models. International Journal for Numerical and Analytical Methods in Geomechanics, 30(13), 1303-1336. DOI: 10.1002/nag.527

METROPROJEKT Prague PLC: 2009-2012, Extension of the line A of Prague's subway. SO 02-29, Airconditioning tunnel.

Niemunis, A. and Herle, L.: 1997, Hypoplastic model for cohesionless soils with elastic strain range. Mechanics of Cohesive Frictional Materials, 2 (4), 279-299.

DOI: $10.1002 /($ SICI)1099-

1484(199710)2:4<279::AID-CFM29>3.0.CO;2-8

Potts, D.M. and Zdravkovič, L.: 1999, Finite element analysis in geotechnical engineering theory. London, $440 \mathrm{pp}$.

Project No. TA04031603: 2014, Integrated tool for practical application of advanced computational models for geomaterials allowing for increase of safety and lifetime of traffic infrastructure. Tech. rep., CTU in Prague, Faculty of Civil Engineering. (http://mech.fsv.cvut.cz/TA04031603/)

Roscoe, K.H. and Burland, J.B.: 1968, On the generalised behaviour of 'wet' clay. Heyman, J. and Lechie. F.A. (Eds.): Engineering plasticity, Cambridge University Press. 535-609.

Svoboda, T., Mašín, D. and Boháč, J.: 2009, Hypoplastic and Mohr-Coulomb models in simulations of a tunnel in clay. Tunel, vol. 18 (4), 59-68.

Svoboda, T., Mašín, D. and Boháč, J.: 2010, Class A predictions of a NATM tunnel in stiff clay. Computers and Geotechnics, 37 (6), 817-825.

DOI: $10.1016 /$ j.compgeo.2010.07.003 